Recherches en didactique des langues et des cultures

Les cahiers de l'Acedle

Dialogisme et discours en situations didactiques

\title{
Dialogisme et spécificités du discours enseignant face à la scolarisation des enfants gitans
}

Nathalie Auger et Sophie Dufour

\section{OpenEdition}

Édition électronique

URL : http://journals.openedition.org/rdlc/1916

DOI : $10.4000 /$ rdlc. 1916

ISSN : 1958-5772

Éditeur

ACEDLE

Référence électronique

Nathalie Auger et Sophie Dufour, « Dialogisme et spécificités du discours enseignant face à la scolarisation des enfants gitans ", Recherches en didactique des langues et des cultures [En ligne], 14-2 | 2017, mis en ligne le 15 juin 2017, consulté le 17 juin 2020. URL : http://journals.openedition.org/rdlc/ 1916 ; DOI : https://doi.org/10.4000/rdlc.1916

Ce document a été généré automatiquement le 17 juin 2020.

\section{$\Theta \Theta \Theta \Theta$}

Recherches en didactique des langues et des cultures is licensed under a Creative Commons AttributionNonCommercial-NoDerivatives 4.0 International License 


\title{
Dialogisme et spécificités du discours enseignant face à la scolarisation des enfants gitans
}

\author{
Nathalie Auger et Sophie Dufour
}

\section{Introduction}

1 Les spécificités du discours enseignant que nous nous proposons d'analyser sont issues d'un corpus recueilli entre septembre 2013 et juin 2014 dans les écoles d'une ville moyenne du sud de la France qui accueillent de nombreux enfants gitans (parfois même exclusivement). L'enquête a été rendue possible par le soutien financier d'un Fonds Social Européen (FSE), en partenariat avec la Direction de l'Action Éducative et de l'Enfance de la Ville et l'Education Nationale. La commande de la recherche portait sur l'« oralité, la langue et les imaginaires » chez les populations gitanes (en particulier les enfants) dans le but d'une meilleure réussite scolaire des élèves. Il se trouve que compte tenu des discours de classe récoltés très marqués par l'absentéisme et les langues et cultures en présence, sujets étroitement corrélés à la scolarisation des enfants gitans, nos analyses se sont révélées comme nous le verrons très intéressantes du point de vue du dialogisme à l'œuvre.

\section{Descriptions du corpus et du public}

\section{Le corpus}

2 Ce terrain peu connu, peu décrit, a dans un premier temps fait l'objet d'enquêtes ethnographiques pendant un an qui ont révélé, au travers des discours enseignants et des travailleurs sociaux de la Ville, des références multiples à l'absentéisme des élèves, à une situation interculturelle inédite (classe composée parfois uniquement d'enfants gitans) et à des questionnements quant aux pratiques scolaires à mettre en œuvre dans ce contexte. 
Ces observations discursives au long court, avant tout qualitatives et ethnographiques, ont orienté notre analyse des interactions qui s'effectue présentement à partir des enregistrements vidéos de classe (cf. infra). En effet, il nous a semblé pertinent d'examiner si ces thèmes récurrents qui semblaient poser question (visibles par exemple dans les entretiens et lors des formations que nous avons pu proposer dans le cadre du projet) étaient repris dans les discours des enseignants et si des conséquences sur leurs pratiques effectives d'enseignement étaient notables. Une de nos préoccupations étant d'ordre éthique, nous cherchons à observer si les discours sont modifiés par les représentations et les discours sur la communauté gitane, et dans quelles mesures elles sont pertinentes dans la situation de classe. Nous nous appuierons, pour le présent article sur un corpus récolté dans la classe d'une école primaire. L'enseignante y était en poste depuis 9 ans. Les élèves de la classe observée ont entre 6 à 7 ans et sont en cours préparatoire.

3 Sur les $4 \mathrm{~h} 30$ d'enregistrement vidéo réalisés, trois extraits d'environ 5 minutes au total sont analysés avec les outils de l'analyse de discours en interaction (C. KerbratOrecchioni, 2005) en s'appuyant sur les phénomènes dialogiques. Si la séance a commencé avec un seul apprenant (extrait 2), ce qui n'est pas inhabituel sur le terrain observé, la journée s'est terminée avec 13 enfants dans la classe.

\section{Caractéristiques du public apprenant : quelques réalités face aux discours}

4 Avant de décrire les interactions verbales collectées en classe, il semble important de rendre compte des résultats les plus saillants obtenus à l'issue d'un an d'enquête ethnographique (Auger \& Matheu, 2016). Tout d'abord, quelques éléments factuels éclairants : les enfants gitans scolarisés sont Français et sédentaires depuis la seconde guerre mondiale, et ils parlent le catalan-roussillonnais. Cette langue ${ }^{1}$ peut s'écrire en reprenant les normes du catalan central (en y adjoignant les graphies nécessaires aux spécificités du roussillonnais). La prosodie (intonation) est la spécificité majeure qui permet la distinction entre gitan et non gitan en catalan-roussillonnais, sans que cela entrave la communication ou l'intercompréhension. «Le gitan» est une langue qui est doublement minorée car, si elle est la langue de la population gitane, elle présente de surcroit une variation du catalan (catalan roussillonnais) qui apparait comme une forme « abâtardie » du catalan, sans écriture. Pourtant, J. Cummins (2001) avance que passer du temps dans une langue minoritaire (familiale, régionale, religieuse) n'entraîne pas de conséquences négatives pour le développement des compétences académiques dans la langue dominante (scolaire, sociale et extra-familiale). Le gitan ${ }^{2}$ n'est souvent pas considéré comme une ressource (ni du point de vue des familles, ni des enseignants) pour développer les compétences en français oral et écrit. Pourtant, depuis les années 1960, des travaux (Cummins) mettant en valeur le travail d'activation des transferts de compétence d'une langue à l'autre, l'intercompréhension entre langues parentes ou encore les pratiques consistant à mettre en relation l'oral et l'écrit ont essaimé. Les enquêtes (par entretiens et observation directe) infirment le stéréotype selon lequel la communauté gitane est dans une situation sacralisant l'oral qui entraverait l'entrée dans l'écrit en français. Ainsi, celle-ci vit avec son temps et s'intéresse aux nouvelles technologies dans les différentes langues parlées/écrites (français, catalan, espagnol notamment) : blogs, réseaux sociaux, textos, visionnage de films sous-titrés ou non. Les 
locuteurs parlent par ailleurs le français sans réaction de rejet. Ils alternent les langues selon les interlocuteurs. Ils sont dans une situation de diglossie (une langue en famille, une autre à l'école). Pour des raisons psycho-affectives évidentes, les parents élèvent leurs enfants dans la langue familiale, mais la communauté gitane regarde la télévision en français, catalan et espagnol. On voit la continuité dans les langues romanes pratiquées qui pourraient être un avantage pour l'Ecole et le développement du français. Par ailleurs, la situation socio-économique difficile dans laquelle se trouve cette population, le repli sur sa communauté pour y trouver l'entraide et échapper à la stigmatisation sont fort bien décrits par la sociologie (cf. Bourdieu 1979, par exemple) et repris dans les documents et les études menées notamment par la Mairie de Perpignan (voir l'ouvrage Cas d'Ecole, le DVD; le rapport Klein, 2010: 14933). Bien que l'absentéisme des enfants reste important, nos enquêtes montrent cependant une entrée dans la scolarisation de plus en plus précoce grâce à la mise en place de centres pré-scolaires, de maternelles de proximité et à un changement de discours de certains parents ${ }^{4}$ qui ont des ambitions de réussite professionnelle pour leurs enfants.

\section{Les discours de la classe : un corpus pour le dialogisme}

5 Si les échanges scolaires peuvent assez communément être étudiés du point de vue dialogal, c'est à dire dans le fil du propos, à travers l'alternance des tours de paroles, entre continuité et rupture, ils peuvent également, et c'est ce qui a attiré notre attention dans ces recherches, l'être du point de vue dialogique (Delarue-Breton, 2014). Cet angle d'approche suppose l'existence d'une interaction entre ce qui est énoncé au fil du propos et d'autres énoncés proférés ailleurs par les enseignants, les familles, les enfants, les médias, avec lesquels sont entretenues des relations de nature variable.

\section{Un corpus pour quels phénomènes dialogiques... ?}

Comme nous l'avons évoqué, le caractère spécifique du contexte éducatif sur lequel portent nos recherches permet selon nous d'interroger une notion telle que le dialogisme, qui pourra éclairer et permettre de décrire la complexité discursive présente dans le corpus recueilli. Cet «outil d'analyse " permet de comprendre les difficultés d'élèves confrontés à la pluralité des voix qui «feuillètent le discours scolaire » (Delarue-Breton, 2014: 23, vol 1). Delarue-Breton a d'ailleurs su mettre au jour l'importance de ces échanges dialogués scolaires, de ce

« dialogisme sans doute interne à la classe, renvoyant à des habitudes de travail où la parole des élèves, leur expérience antérieure et les représentations qui en découlent, tout en devant être dépassées, ont leur place. » (2014:92)

7 Assez «classiquement» on trouve dans notre corpus des marques dialogiques assez prototypiques étudiées par d'autres chercheurs, notamment Delarue-Breton (2014, 2016b), pour les discours de la classe et Marlot et Baques (2013) pour les cours magistraux. Pour rappel, reprenant la tripartition de Bres (2005), Marlot et Baques (ibid.) décrivent ainsi le dialogisme dans le cadre du cours magistral :

- Le dialogisme interdiscursif : Le locuteur (le professeur) fait référence à des propos qui ne sont pas les siens et les mêle à son discours : on note, d'une manière plus ou moins explicite, la présence de la parole d'autrui dans son discours. 
- Le dialogisme intralocutif: Le locuteur (le professeur) est son premier interlocuteur. La production de sa parole se fait constamment en interaction avec ce qu'il a dit antérieurement, avec ce qu'il est en train de dire et ce qu'il a à dire.

- Le dialogisme interlocutif: Le discours du professeur se construit selon les énoncés des étudiants. Cette construction se révèle dans des formes langagières qui prennent en compte l'aspect lexical, syntaxique ou encore la structure argumentative. l'instar de Bres ${ }^{5}$ que la notion de dialogisme a toute sa pertinence pour l'analyse des discours de la sphère d'activité de l'enseignement, tant ceux des enseignants que ceux des élèves et étudiants, élargissant donc le cadre d'une activité dialogique à des situations où les interactants dialoguent véritablement, co-construisent le fil de ce discours tenu en classe (ibid.). l'enseignant, intéressées par les phénomènes dialogiques qui entrent en jeu le plus souvent dès lors qu'il corrige les productions des élèves sur la forme ou le fond (dialogisme interlocutif), reformule sa propre parole (dialogisme intralocutif ${ }^{6}$ ), ou fait références à d'autres discours, d'autres séances (visibles sous la forme de marques dialogiques interdiscursives). C'est essentiellement ce dernier type de dialogisme, massif dans notre corpus, qui a attiré notre attention, bien qu'il nous faille préciser l'acception que nous lui attribuons en fonction des observations faites.

effet, dès lors que l'on problématise dialogisme et terrain "dialogué », il apparait important de préciser dans quel cadre nous le faisons. Nous proposons d'adapter en quelque sorte la définition précédente du dialogisme interdiscursif, à un cadre énonciatif plus large puisqu'il semble difficile qu'un enseignant en classe puisse clairement dissocier ses propos de ceux qui ont été construits collectivement ${ }^{7}$. Pourrait donc apparaitre ce que nous nommerions un dialogisme interdiscursif « scolaire » dont la définition serait la suivante :

- Le locuteur (le professeur) fait référence dans son discours à d'autres discours de la classe qu'il a peu ou prou aidé à construire.

11 Si ces observations semblent plus ou moins transférables à la plupart des situations de classe, il nous faut ici clairement marquer ce qui fait la spécificité du terrain observé. Les traces d'interdiscursivité relevées dans le discours de l'enseignante de notre corpus seront bien entendu les marques d'interventions à valeur de rétroactions convoquant d'autres savoirs/discours, et cela afin de mener à bien l'apprentissage. Cependant, nous verrons ensuite qu'en s'attachant sans cesse à créer du lien avec les discours tenus dans les séances précédentes, en tenant des propos très individualisés envers les élèves de la classe, celle-ci traduit dans ses paroles ce qui caractérise la singularité de la situation d'enseignement observée, à savoir le manque d'assiduité des élèves ainsi que la forte prégnance des langues et cultures de son public.

\section{Activités correctives et dialogisme interlocutif}

12 La bifocalisation "naturelle (Bange, 1996) de tout échange exolingue et ici plus spécifiquement du discours enseignant, s'exprime au travers de pratiques discursives qui semblent à chaque fois renvoyer à une norme linguistique, mais aussi potentiellement aux contenus de discours tenus antérieurement et sur lesquels s'appuie l'étayage correctif

Recherches en didactique des langues et des cultures, 14-2 | 2017 
de l'enseignant. Ainsi, dans l'exemple tiré de notre corpus, l'enseignante reprend - une fois de plus - cet apprenant qui confond « pourquoi » et « parce que » :

\section{Exemple 1}

Maitresse : pourquoi fait-il cela $\uparrow$

Elève : pourquoi ça fait

Maitresse : parce que / parce que ça fait

On observe donc que l'hétérocorrection : parce que ça fait s'attache d'une part à corriger un syntagme défaillant, mais que ce faisant l'enseignante s'adresse aussi plus directement à son interlocuteur dont elle connait les difficultés propres. Ce type d'intervention semble réellement connecté à un vécu conversationnel commun, plus loin l'enseignante dira à un apprenant : tu t'en rappelles toi de ta difficulté, il s'agit précisément de cela, du fait qu'elle est constamment témoin de tout ce qui se dit et que sa "mémoire vive » stocke tous les discours énoncés en classe. Une autre stratégie visant à corriger un apprenant peut consister à orienter ses productions afin de l'amener à formuler lui-même des éléments attendus de réponse. Ainsi dans ce passage :

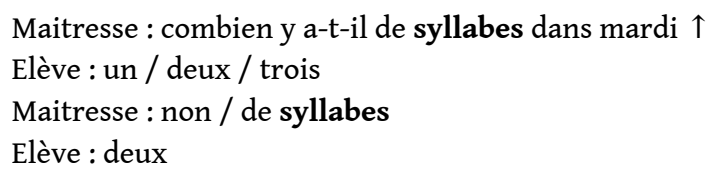

Cette fois c'est le contenu qui semble poser problème, le sens du mot «syllabe » n'étant pas remobilisé instantanément par l'apprenant. L'enseignante choisit donc de baser son intervention sur la répétition de ce mot (non/ de syllabes) afin de permettre à son interlocuteur de pointer ce qu'elle attend en retour, faisant le pari qu'il en a les moyens puisque cela a déjà été vu en classe.

Pour illustrer encore cet aspect propre à un dialogisme en situation scolaire, voici des exemples à valeur de rappel de savoirs censés être stabilisés antérieurement, et supposés servir d'étayage pour la compréhension de nouvelles acquisitions :

- qui c'est qui chante comme ça / le s9 $\uparrow$ (comprendre : «nous avons vu que le 's' chantait, sifflait »)

- après la consonne tu as quoi $\uparrow$ une consonne $\uparrow$ non (comprendre : « nous avons vu les doubles consonnes »)

- ne dis pas n'importe quoi / c'est le Père Noël en ce moment $\uparrow$ (comprendre : «nous avons vu les saisons et les fêtes associées, et en ce moment ce n'est pas la saison du Père-Noël »)

Dans chacun de ces cas, l'enseignante fait référence implicitement à des discours déjà tenus ou des objets communs expliqués en classe: le « $s$ » chante, les élèves savent reconnaître les consonnes et au printemps ce n'est pas la saison du Père Noël. Ces occurrences sous la forme de dialogisme interdiscursif sont également des questions didactiques (Boissat, 1991): l'enseignant connaît la réponse et en proposant une 
référence à des savoirs rencontrés dans le passé espère que l'élève va pouvoir répondre à sa question.

Plus spécifiquement, d'autres occurrences font référence au métier d'élève (Perrenoud, 1994), cette compétence développée dès l'école maternelle afin d'entrer dans les apprentissages et le vivre ensemble dans le groupe classe. En voici quelques exemples :

- tiens-le (stylo) correctement / chaque fois tu me le tiens n'importe comment (comprendre : « je t'ai déjà montré comment tenir le stylo »)

- allez c'est pas la première fois que vous écrivez dessus (sur le Tableau Blanc Interactif) (comprendre : «je vous ai déjà montré comment écrire sur le TBI »)

- non fais un espace (entre les mots en écrivant sur le TBI) / tu sais bien / non / non (comprendre : «je vous ai déjà montré comment faire un espace »)

Ces différentes traces d'un dialogisme interdiscursif tel que défini, ancré sur un terrain scolaire, ont aussi valeur assez classiquement de stratégies destinées à remobiliser des savoirs, à rappeler des consignes maintes fois répétées qui devraient être actualisées dans les pratiques selon l'enseignante. Quel que soit leur degré d'explicitation, nous aurions pu encore proposer d'autres exemples de rétroactions correctives (Lyster, 1999) tirées de notre corpus, celles-ci auraient invariablement comporté des références supposées à une norme et à des discours tenus « sur ».

\section{Des formes de dialogisme interdiscursif plus spécifiques à la scolarisation des enfants gitans}

Dans notre corpus, les formes de dialogisme précédemment discutées sont omniprésentes. Si les pratiques discursives décrites semblent ainsi soulever des problématiques communes aux situations d'enseignement telles que l'on peut les rencontrer habituellement, il semblerait que les contours d'un dialogisme spécifique se dessine, conséquent de l'absentéisme des élèves ainsi que de leur vécu extra-scolaire et linguistique.

\section{Effets de l'absentéisme sur la conduite de la classe}

Nous proposons donc à l'étude un premier extrait de corpus qui illustre de façon assez prototypique, des observations ethnographiques que nous avons effectuées sur le terrain pendant un an.

19 Si le dialogisme interdiscursif tel que nous l'avons précédemment défini (cf. supra) est présent de façon massive dans nos données, c'est qu'il traduit chez l'enseignante une volonté de contextualiser sans cesse ses propos en fonction d'autres discours qu'elle a pu tenir antérieurement et auxquels les élèves n'ont pas tous assisté. Il est question ici de la difficulté à mémoriser/accumuler des savoirs lorsque l'on est absent. À l'instar de Delarue-Breton (2014:55), nous considérons en effet que :

...loin d'être le produit de la seule alternance des tours de parole, le dialogue scolaire convoque aussi d'autres énoncés qui se répondent, les textes de savoirs. De plus, ces énoncés produits dans la classe, au-delà de référer plus ou moins implicitement à des énoncés produits ailleurs et dans un autre contexte, s'enracinent aussi dans les séances antérieures...

L'enchaînement des séquences pédagogiques entre elles crée donc une cohérence que peut rompre le manque d'assiduité des élèves. L'extrait suivant entend illustrer cette problématique : 
1. Houcine : il comprend pas / hein (en parlant d'Isaac)

2. Atsem : il comprend pas parce qu'il était pas là hier Houcine / toi tu comprends parce que t'étais là hier

3. Houcine : oui

4. Atsem : comme ça il va le savoir aussi / il va savoir ce que c'est

5. Maitresse : allez Kelly je vais t'expliquer quelque chose // regarde / en fait / j'ai / hier / tu sais j'ai expliqué Houcine en fait il a travaillé sur l'ordinateur / il a écrit / mais il a écrit en fait en utilisant une police / qui ressemble au moyen-âge tu sais comme les châteaux forts / donc ici il va falloir maintenant / c'est comme un petit jeu il faut que tu trouves ton prénom écrit comme les chevaliers

6. Houcine : je sais pas où il est $\uparrow$

7. Maitresse : toi tu es dans ce groupe / cherche-le ton prénom écrit comme les chevaliers

8. Kelly : dans ce groupe

9. Maitresse : oui dans ce groupe / regarde bien Kelly / tu vas le trouver j'en suis sûre / et il est écrit avec des lettres minuscules / c'est pas des majuscules ce ne sont pas des majuscules / bonjour (échange à voix basse avec l'Atsem) // ah oui / est-ce que je peux vous voir à midi moins le quart s'il vous plait $\uparrow$ merci (s'adresse à une maman qui vient d'entrer dans la classe)

10. (échanges en catalan-roussillonnais entre les enfants)

11. Maitresse : alors Kenzo / hier Houcine / comme il était tout seul / il a eu le droit d'aller à l'ordinateur / et il a écrit tous les prénoms de la classe / avec une police médiévale c'est-à-dire une écriture qui ressemble au moyenâge / à l'époque des chevaliers / donc il faut que t'ailles chercher ton étiquette et que tu retrouves ton prénom

12. Houcine intervient et montre les étiquettes à ses camarades

13. Maitresse : ne le dis pas Houcine / c'est un jeu /sinon il pourra pas jouer / viens t'asseoir Houcine / s'il te plait // du coup j'ai même pas fait la date / j'ai même pas fait / j'ai pas fait le programme de la / pas grave on va le faire maintenant / allez

Il est ici essentiellement question de la difficulté à suivre la progression pédagogique si l'on est absent. Ce constat renvoie à de nombreux entretiens dans lesquels les enseignants nous disent ne pas pouvoir construire de progression du fait de l'absentéisme des enfants. Leur propre statut d'enseignant est ainsi mis en difficulté par l'absence fréquente de public.

D'entrée de jeu dans cet extrait, la négation dialogique il comprend pas au tour 1 produite par un élève (Houcine) concernant un de ses camarades, montre que les élèves euxmêmes savent que l'objectif des séances de classe est la compréhension afin de pouvoir entrer dans les activités prévues. L'Atsem ${ }^{11}$ propose une explication à cela en opérant une dichotomie (manifeste par la pause) : il (Isaac) comprend pas parce qu'il était pas là hier Houcine / toi tu comprends parce que t'étais là hier. L'opposition s'atténue lorsque celle-ci, en utilisant une forme du futur proche, explique que ce problème va être résolu et que l'élève absent pourra rentrer dans le groupe de ceux qui ont compris : comme ça il va le savoir aussi / il va savoir ce que c'est.

L'enseignante qui souhaite visiblement passer à un autre sujet s'adresse à une autre élève au tour 5: allez Kelly je vais t'expliquer quelque chose. Elle reconstruit discursivement la situation de classe vécue la veille pour permettre la compréhension aux absents et ne pas s'appesantir sur la remarque de l'Atsem. Le jeu des personnes (je, tu, il) et de la 
temporalité (hier, maintenant, dans un futur proche) est encore signe d'un renvoi interdiscursif en relation avec le contexte de la séance précédente au tour 5 : je vais t'expliquer... (versus) hier... en fait il a travaillé sur l'ordinateur / il a écrit... / donc il va falloir maintenant... / il faut que tu trouves ton prénom... Dans son propos ici, on remarquera que le discours de l'enseignante est ponctué du marqueur dialogique: tu sais ( 2 occurrences) visant à mobiliser des connaissances antérieures sans pour autant être certaine que ces savoirs soient stabilisés.

Dans cet extrait toujours, l'enseignante semble déstabilisée par le fait que les élèves, audelà des questions d'absentéisme, ne réussissent pas l'activité (retrouver son prénom en écriture caroline). Elle encourage au tour 9: regarde bien Kelly / tu vas le trouver j'en suis sûre, opère une rétro-action négative par rapport à la réponse de l'élève juste après : c'est pas des majuscules ce ne sont pas des majuscules. En reformulant même sous la forme de dialogisme intralocutif sa propre parole pour la rendre plus conforme à la norme de l'écrit : c'est pas... ce ne sont pas des majuscules, elle réaffirme aussi sa posture d'enseignante garante de la norme scolaire.

On observe ensuite une interruption de la séance par un parent qui vient d'entrer en classe amenant son enfant en retard. L'enseignante demande à discuter avec lui quand il reviendra chercher son fils à midi au tour 9 encore (est-ce que je peux vous voir à midi moins le quart s'il vous plait $\uparrow$ merci). Elle peine ensuite à revenir dans l'interaction qu'elle a initiée et, propose, de nouveau, une recontextualisation de l'activité sous la forme interdiscursive au tour 11 : hier Houcine... il a écrit les prénoms de la classe/ avec une police médiévale. Elle espère que cette explication permettra à Kelly de comprendre la consigne ( donc il faut que t'ailles chercher ton étiquette et que tu retrouves ton prénom). L'élève présent à la séance précédente souhaite alors aider ceux qui ne retrouvent pas leur prénom, initiative que l'enseignante corrige au tour 13 : ne le dis pas Houcine / c'est un jeu / sinon il pourra pas jouer.

L'extrait se clôt sur cette remarque : du coup j'ai même pas fait la date /j'ai même pas fait /j'ai pas fait le programme, qui montre bien les répercussions que peuvent avoir ce jonglage permanent entre les uns et les autres sur la conduite d'une routine de classe ${ }^{12}$.

Dans un second extrait, l'enseignante fait classe à un seul élève en début de journée. On voit de quelle manière elle va gérer cette activité programmée d'écriture d'un scénario de conte de fée, déjà commencée « en groupe » :

1. Maitresse : allez on y va / alors Houcine / est-ce que tu te rappelles un petit peu de l'histoire / que vous avez inventée //

2. Houcine : non

3. Maitresse: non tu t'en rappelles pas $\uparrow$ elle va parler de quoi cette histoire

4. Houcine : de chevaliers

5. Maitresse : oui / de chevaliers / et quoi d'autre $\uparrow$

6. Houcine: de princesses

7. Maitresse : ooui / et quoi d'autre $\uparrow / /$ donc les personnages il y a il y aura des personnages dans ce film // donc vous avez dit qu'il y aurait qu'il y aura des chevaliers /

8. Houcine : des reines

9. Maitresse : des reines / des $r$

10. Houcine : des rois

11. Maitresse : quoi d'autre $\uparrow / /$ 


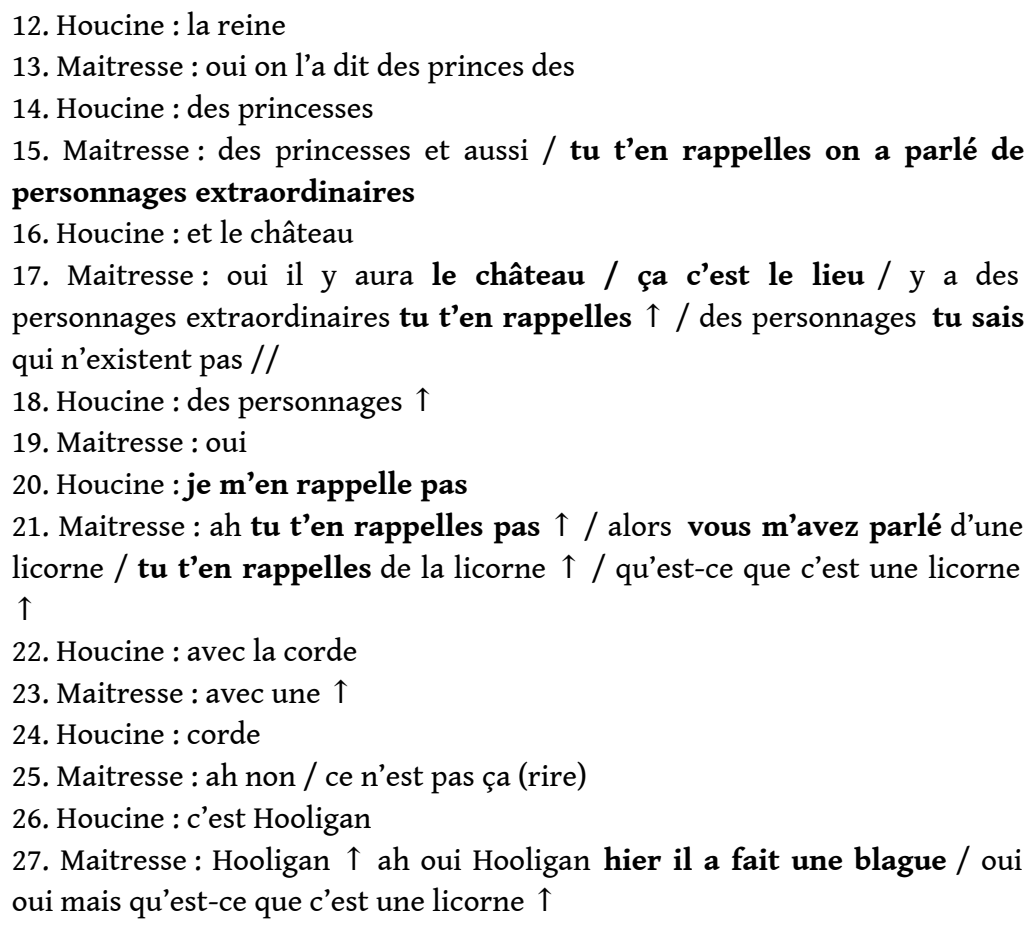

L'enseignante signale le début de l'activité : allez on y va / alors Houcine et mobilise immédiatement des savoirs censés être mémorisés de la séance précédente : tu te rappelles un petit peu de l'histoire. Là encore, le jeu des pronoms au tour 1: tu/vous (on) comme dans tu te rappelles... l'histoire/ que vous avez inventée manifeste le mouvement complexe de mémorisation entre ce qui a été imaginé par un collectif (l'histoire / que vous avez inventée... vous avez dit... on a parlé... alors vous m'avez parlé) et sa réactivation faite individuellement afin de reprendre le fil du travail de classe et des échanges de l'extrait : tu te rappelles... tu t'en rappelles pas... tu t'en rappelles... je ne m'en rappelle pas... ah tu t'en rappelles pas... tu t'en rappelles de la licorne. Au fil des réponses données par l'élève concernant les personnages puis le lieu, l'enseignante tente de réactualiser le schéma de Propp (1970) (aux tours 7, 15 et 17 : donc les personnages... on a parlé de personnages extraordinaires... le château / ça c'est le lieu) sans vraiment que cette structure ne semble faire sens pour l'élève.

On observera que les phénomènes de dialogisme liés à la recontextualisation appuyée due à l'absentéisme et à l'instabilité des connaissances construites par les élèves de la classe, donnent lieu à 3 types de réorganisation impactant largement la progression de la séance ${ }^{13}$, à savoir:

- mais ceux qui ne sont pas venus ce matin iront pas aux ateliers (=réorganisation des activités)

- maintenant que tu viens beaucoup à l'école tu as changé de groupe / oh je l'ai peut-être dit à ta maman et pas à toi (=réorganisation des groupes)

- oui parce qu'il n'était pas là toi tu étais là / je vais lui expliquer maintenant (=réorganisation des modalités explicatives selon les absences/présences).

Ces occurrences sont également parfois symptomatiques de l'agacement ${ }^{14}$ (ceux qui sont pas venus iront pas), de la confusion (oh je j'ai peut-être dit à ta maman et pas à toi), des appréciations (c'est dommage... c'est pas grave...) qui prévalent dans la classe et qui sont en relation avec les absences des élèves comme dans les exemples ci-dessous :

- c'est dommage $\mathrm{X}$ que tu ne sois pas arrivé il y a une demi-heure et on a travaillé sur les chevaliers / bon c'est pas grave (comprendre : « tu aurais dû arriver il y a une demi-heure ») 
- je vais choisir K. parce qu'il a eu le courage de venir ce matin déjà (pour être chevalier) (comprendre : «d'autres n'ont pas eu ce courage »)

- moi aussi je veux être reine / eh bah viens le matin ma pauvre (comprendre : «tu devrais venir le matin si tu veux participer aux activités »)

- tu t'en rappelles $\uparrow$ t'étais là / je l'ai marqué (comprendre: «tu devrais t'en souvenir puisque tu étais là »)

Nous avons vu comment le discours enseignant s'accommode de la complexité de ce terrain inédit d'enseignement où la gestion d'un groupe-classe ne peut être envisagée que de manière "individualisante ", puisque l'enseignante a en face d'elle des interlocuteurs dont elle n'est jamais sûre qu'ils aient tous le même préconstruit, la même " histoire conversationnelle » dans la classe.

\title{
Procédés dialogiques en relation avec les langues et les cultures des enfants
}

Nous renvoyons de nouveau aux travaux de Delarue-Breton (2016b) qui mettent en exergue l'importance de ce que Jodelet (2006) nomme le préconstruit culturel, qui souligne que les significations produites par les individus dans un contexte donné s'inscrivent également dans l'expérience antérieurement vécue, entendue comme socialement définie et construite. Cet élément semble tout à fait pertinent concernant nos élèves, qui nous l'avons évoqué, n'ont pas le français comme langue familiale. Apparaissent dans les discours tenus en classe, des traces évidentes des langues et cultures en présence ainsi que des occurrences dialogiques qui témoignent de représentations, de paroles rapportées existant en dehors de la classe. Les deux extraits qui suivent (3 et 4$)$ sont très représentatifs selon nous du type d'échanges qui circulent dans la classe de l'enseignante observée.

Dans ce passage, elle projette sur le tableau blanc interactif une photo masquée en partie de l'artiste italien du $16^{\text {ème }}$ siècle, G. Arcimboldo (Les saisons). Il s'agit pour elle de faire découvrir progressivement ce portrait composé de fruits et de légumes aux élèves afin de susciter leur curiosité.

\section{Extrait 3}

\author{
1. Elève : c'est un payo ${ }^{15}$ \\ 2. Maitresse : pardon $\uparrow$ \\ 3. Elève : c'est un monsieur \\ 4. Maitresse : c'est un monsieur bah pourquoi tu me dis ça $\uparrow$ je vois pas de \\ monsieur moi \\ 5. Elève : regarde les oreilles et \\ 6. Maitresse : ah bon \\ 7. Elève : les cheveux \\ 8. Maitresse : mais moi je vois du raisin \\ 9. Elève 1 : regarde c'est un payo regarde \\ 10. Elève $2:$ c'est un homme \\ 11. Maitresse : et c'est pas un payo / c'est un gitan // es un gitana ${ }^{16}$ (rire) // \\ vous voyez tous un monsieur \\ 12. Elève : non / c'est une statue (elle affiche la photo en entier) \\ 13. Maitresse : bon / on va pas dire payo / on va dire un homme / pourquoi il \\ serait français $\uparrow$ il serait pas italien ou espagnol $\uparrow$
}

Alors que le tableau semble dévoiler un personnage, un élève utilise le mot " payo » qui signifie en catalan roussillonnais «non-gitan». Il est intéressant de voir que ce 
phénomène que l'on pourrait considérer comme étant assez classique chez les allophones, assimilable à de l'alternance codique permettant de dire en langue maternelle un mot non connu en français, n'en n'est pas vraiment, car les élèves au tour 3 (c'est un monsieur) sont en réalité tout à fait capables de nommer en langue cible ce qu'ils entrevoient. Le vocable utilisé, " payo », convoque donc avec lui un discours qui semble d'un autre ordre, qui se déplace sur un terrain plus identitaire. Les répliques de l'enseignante au tour $11:$ et c'est pas un payo / c'est un gitan // es un gitana (rire) // vous voyez tous un monsieur et au tour 13 : bon / on va pas dire payo / on va dire un homme / pourquoi il serait français $\uparrow$ il serait pas italien ou espagnol $\uparrow$ font écho à cela. Elle file la métaphore selon laquelle ce personnage pourrait être gitan, un homme et italien ou espagnol afin d'ouvrir la catégorisation de ce monsieur, mais utilise pour ce faire notamment leur langue maternelle (es un gitana), se plaçant ainsi sur un terrain commun.

Sur le plan interdiscursif, on retrouve dans les propos échangés les traces de discours tenus hors de l'établissement sur la communauté gitane: d'accord ils sont français ben // mais bon /// ils sont pas intégrés quoi // ils sont un peu comme des étrangers ${ }^{17}$, ou par ellemême : oui /// nous on est pas des payo // on est des gitans ${ }^{18}$. Au niveau familial, on ajoutera que les alliances sont souvent endogamiques et qu'au niveau individuel, l'identité qui prévaut est l'appartenance à la communauté gitane. Les représentations en miroir «nous / eux » semblent structurer les représentations des deux communautés (gitane vs non-gitane) nommés et identifiés sous l'appellation : « payo » vs « gitan » qui renvoie à la communauté ou à la langue.

\title{
Dialogisme avec les connaissances linguistiques des enfants
}

Dans nos enregistrements, l'enseignante permet, voire encourage, l'utilisation de la langue maternelle des enfants comme une ressource pour les aider à réaliser des tâches scolaires, et cela bien que l'institution, nous confie-t-elle, n'approuve pas particulièrement ce type de pratique. Après 9 ans passés au contact de ce public, elle comprend très bien ce que les enfants se disent entre eux, cette compétence lui permettant de trouver un terrain privilégié pour les amener vers la langue-cible comme dans ces deux extraits :

\section{Exemple 3}

Elève : c'est du fousta

Maitresse : alors comment on dit en français du fousta $\uparrow$

Elève $1: \mathrm{du}$ bois

Elève $2: \mathrm{du}$ bois

\section{Exemple 4}

\author{
Elève : c'est une gall \\ Maitresse : c'est quoi / comment tu le dis toi en français $\uparrow$ \\ Eleve : un poulet \\ Maitresse : oui (rire) un poulet
}

Le rire dans le second extrait rend bien compte de la complicité qui lie l'enseignante à ses élèves qui, dans un climat apaisé, peuvent ainsi faire valoir leur capacité à passer d'une langue à l'autre. Aussi, si la traduction de certains mots semble finalement assez classique dès lors qu'un enseignant partage la langue maternelle de ses élèves allophones, le fait d'encourager son recours est moins systématique dans les pratiques de classe. Ici on 
observe qu'il s'agit même d'une stratégie d'enseignement/apprentissage comme l'illustre ce passage :

\section{Extrait $4^{19}$}

Maitresse : je ne comprends pas Kélian

Elève : ça c'est des feuilles

Maitresse : c'est pas des feuilles // ce sont des plumes

Elève : mais oui / mais les plumes il fait des feuilles il comprend pas

Maitresse : non je ne comprends pas alors explique-le à quelqu'un en gitan

et euh comme ça vous allez vous allez essayer de trouver d'autres mots

L'idée étant dans cet extrait essentiellement de lever le voile sur une incompréhension qui n'est pas terminologique, mais qui repose bel et bien sur l'interprétation que l'élève se fait de ce qui est représenté sur une image (il voit un arbre là où l'enseignante voit des oiseaux avec des plumes). La stratégie qu'elle propose à son interlocuteur (« explique-le à quelqu'un en gitan ») est la collaboration avec ses pairs (« euh comme ça vous allez vous allez essayer de trouver d'autres mots»), faisant de nouveau référence à une appartenance linguistique commune.

\section{Extrait $5^{20}$}

1. Maitresse : non / je vous demande ce que tu, je vous demande de décrire ce tableau alors //

2. Elève : $a h$

3. Maitresse : alors // et ce que tu vois ici / comment ça s'appelle $\uparrow$

4. Elève : là

5. Maitresse: une châtaigne $\uparrow$ comment vous le dites vous le mot châtaigne $\uparrow$

6. Elève : une euh châtaigne

7. Maitresse : c'est pas une castaña $\uparrow$

8. Elève : euh si

9. Maitresse : voilà là vous avez une châtaigne

Le pronom "vous " répété au tour 5 est riche de sens car il renvoie dialogiquement au "vous groupe-classe», mais aussi à ce "vous qui parlez une autre langue». Ici l'enseignante en leur donnant le terme " châtaigne », cherche de plus à s'assurer qu'il est bien compris en ayant recours à la traduction (comment vous le dites-vous le mot châtaigne $\uparrow$ ), mais un des élèves de manière inattendue répète le mot en français (une euh châtaigne). Il semble qu'il ne prenne pas en compte le second «vous » évoqué cidessus, l'enseignante se voit donc obligée d'expliciter son intention avec cette proposition "c'est pas une castaña $\uparrow$ ", menant ainsi à bien son entreprise en reprenant "voilà là vous avez une châtaigne » après qu'un apprenant lui a répondu.

Les quelques pratiques à visée pédagogique observées montrent clairement que l'enseignante, en convoquant dans la classe une autre langue que la langue-cible, entend entériner le fait qu'il s'agit pour elle d'une ressource disponible pour aider à l'accès au sens et à l'apprentissage. Les propos recueillis juste après la séance filmée directement auprès d'elle soulignent sa volonté de valoriser la langue et la culture des enfants ${ }^{21}: c^{\prime} e s t$ une richesse, à chaque fois je lie ces évènements-là (Noël, la galette des rois, etc.) à leur culture / et après à la culture dans le monde, je veux leur montrer que moi leur culture je la trouve belle / et y a des choses à savoir et c'est important / et du coup ils sont contents ils se sentent reconnus. Cette attitude montre que le dialogue permanent qu'elle engage avec ce qui se passe dans la 
classe et ce que sont et vivent ses élèves en dehors de celle-ci, relève d'une dynamique qui prend en compte les spécificités de son public dans toute ses dimensions.

\section{Conclusion}

43 L'étude du dialogisme enseignant de notre corpus nous a permis de rendre compte discursivement de "malaises » liés à un sentiment d'échec souvent décelés dans les paroles des enseignants lors d'entretiens ethnographiques réalisés antérieurement à nos analyses (cf. supra). Les enseignants évoquaient très souvent en effet leurs difficultés à gérer, faire progresser les élèves du fait de leur absentéisme. Leur rôle, leur mission d'enseignant se trouvait aussi mise à mal: Quelle est la raison d'être de mon travail si mes élèves ne sont pas/peu présents?, sans compter l'atteinte à la face selon Goffman (1974): On ne me considère pas dans mon rôle, je ne suis pas pris au sérieux. Ces considérations renforçaient la perception que ces difficultés provenaient sans aucun doute de la culture et de la langue gitanes...

L'étude des marques du dialogisme interdiscursif scolaire de notre corpus vidéo montre au contraire que les éléments langagiers et culturels peuvent permettre d'introduire d'autres pratiques et, $a$ fortiori, d'autres représentations des uns et des autres invitant enseignants et élèves à travailler, à co-construire. La manière dont l'enseignante observée conjugue les discours produits hors et dans la classe est donc à ce titre très intéressante. Par ailleurs, l'analyse discursive effectuée nous a également permis de comprendre les répercussions de l'absentéisme sur les pratiques de classe et nous amène à différentes considérations didactiques. La pédagogie différenciée, préconisée par les instructions officielles de l'Education Nationale, est donc plus que jamais d'actualité dans cette situation. Ainsi, le travail sous forme de séquence avec un objectif de tâche finale étant certainement difficile à réaliser dans les conditions décrites, on peut suggérer qu'une approche par unités d'apprentissage sous forme de séances autonomes permettrait de se libérer d'un renvoi permanent aux séances précédentes. Dans la mesure où une progression reste forcément indispensable, nous pourrions l'envisager sous la forme d'un portfolio suivant chaque élève qui rendrait compte de ses acquis et des compétences en cours d'acquisition, ou restant à développer. Ces observations sont de l'ordre d'une didactique ascendante : les contextes peuvent inspirer des façons alternatives d'enseigner et réinterroger des conceptions didactiques.

\section{BIBLIOGRAPHIE}

Auger, N. \& Matheu, N. (2016). « Du gitan au français : d'une langue hors-norme à une langue normée ? ». In Collette, K et Steuckardt, A. (dir.) : Discours hors norme, constructions sociales. Signes, discours et société, n¹6. http://www.revue-signes.info/document.php?id=4721.

Bange, P. (1996). « Considérations sur le rôle de l'interaction dans l'acquisition d'une langue étrangère ", Les Carnets du Cediscor. 4/1996. pp. 189-202. http://cediscor.revues.org/443. 
Boissat, D. (1991). «Questions de classe : question de mise en scène, question de mise en demeure ». In Kerbrat-Orecchioni. C. (dir.) : La question. Presses Universitaires de Lyon. pp. 263-294.

Bourdieu, P. (1979). La distinction : critique sociale du jugement. Paris : éd. de minuit.

Bres, J. (2005). « Savoir de quoi on parle : dialogue, dialogal, dialogique ; dialogisme, polyphonie... ». In Bres, J., Haillet, P.-P., Mellet, S., Nolke, H. \& Rosier, L. (dir.). Actes du colloque de Cerisy. Dialogisme et polyphonie. Approches linguistiques. Bruxelles : De Boeck-Duculot. pp. 47-61.

Bronckart, J.-P. (2016). Pourquoi et comment devenir didacticien?, coll. Savoir mieux. Lille : Presses Universitaires du Septentrion.

Carrère, (2009). « Scolarisation, éducation et familles d'origine gitane - Perpignan », Diversité, $\mathrm{n}^{\circ}$ 159, page 108 (09/2009) http://www.educ-revues.fr/DVST/AffichageDocument.aspx?iddoc=39291

Cummins, J. (2001). Negotiating identities: Education for empowerment in a diverse society. $2^{\text {nd }}$ Edition. Los Angeles : California Association for Bilingual Education.

Delarue-Breton, C. (2014). « Dialogue scolaire, dialogisme et évènements d'énonciation : malentendus et in-entendus au sein de la classe ». Études de Linguistique Appliquée, n¹73. pp. 87-97.

Delarue-Breton, C. (2016a). Inégalité d'accès au savoir, ou inégalité d'accès au questionnement? De l'étude du dialogisme du discours scolaire à l'étude de l'activité dialogique des élèves et des étudiants. Volume II. Note de synthèse, 220 pages.

Delarue-Breton, C. (2016b). « Le dialogal et le dialogique: quelques remarques sur les heurs et malheurs de l'interaction verbale en classe de français ». In Neveu, F., Bergounioux, G., Côté, M.H., Fournier, J.-M., Hriba, L. \& Prévost, S. (Éds.). Actes du Vème Congrès Mondial de Linguistique Française (CMLF), Symposium « Linguistique et didactique ».

Goffman, E. (1974). Les rites d'interaction. Paris : Minuit.

Habermas, J. (1987). Théorie de l'agir communicationnel. 2 vol. Paris: Fayard.

Huguet, J. (2012). Cas d'école. Scolariser les enfants gitans sédentaires de Perpignan. Les ateliers du passeur.

Jodelet, D. (2006). « Place de l'expérience vécue dans le processus de formation des représentations sociales ». In Hass, V. (dir.). Les savoirs du quotidien. Transmissions, Appropriations, Représentations. pp. 235-255. Rennes : PUR. Collection : Didact - Psychologie sociale.

Kerbrat-Orecchioni, C. (2005). Le discours en interaction. Paris : A. Colin

Lyster, R. (1994). « La négociation de la forme : stratégie analytique en classe d'immersion ». The Canadian Modern Language Review, ${ }^{\circ} 50$. pp. 447-465.

Marlot, C. \& Baques, M.-C. (2013). Le dialogisme à l'œuvre dans le cours magistral à l' université : un point de vue didactique, Apprendre et former : la dimension langagière. Presses Universitaires BlaisePascal. www.lcdpu.fr, pp. 359-394, Sphère éducative, 978-2-84516-584-7. <halshs-00976327>

Perrenoud, P. (1994). Métier d'élève et sens du travail. Paris : ESF.

Propp, V. (1970). Morphologie du conte. Paris : Seuil, coll. « Points / Essais ». 


\section{NOTES}

1. Cf. notre étude GypsyLang en ligne https://www.ortolang.fr/market/corpora/prax000931.

2. Donc le catalan-roussillonnais.

3. Nous ne reviendrons pas sur la présentation des quartiers et de la population disponibles dans l'ouvrage Cas d'école, le rapport de l'Education de 2008 par exemple. Rappelons simplement que la population gitane de l'étude compte de 4000 à 7000 personnes, sur une population totale de 115000 habitants (Carrère, 2009) répartie sur des quartiers ségrégués, dont celui qui accueille l'école dans laquelle nous nous sommes rendues.

4. Durant les enquêtes évoquées, ont pu être recueillies des paroles telles que J'aimerais bien que ma fille travaille à la banque ou en coiffure, Maintenant que mes enfants sont à l'école j'ai du temps, J'aimerais retourner à l'école aussi.

5. Cf. son article dans le présent numéro, « Dialogisme, éléments pour l'analyse », chap. 6.

6. Le dialogisme intralocutif s'avère très difficilement identifiable dans notre corpus, marqué par le cadre participatif de la classe, dialogal par essence.

7. En didactique, le passage de savoirs mondains (l'expérience du monde, les expériences des élèves, Delarue-Breton, 2016a) aux savoirs savants s'effectue par la négociation interactionnelle (cf. les travaux socioconstructivistes dans la lignée d'Habermas, 1987, et de Bronckart, 2016).

8. Conventions de transcription: / pause brève, // pause longue, $\uparrow$ intonation montante, chevauchement.

9. L'enseignante se réfère ici à la méthode de lecture ludique La planète des alphas (http:// planetedesalphas.ca/) où chaque lettre représente un animal ou un objet, ici dans l'exemple, c'est la lettre « s » qui apparait sous fourme de serpent qui chante, qui siffle.

10. L'enseignante dans cette séance veut que chaque enfant reconnaisse l'étiquette sur laquelle son prénom est écrit en lettres avec une police de style « médiéval ».

11. Agent territorial spécialisé des écoles maternelles.

12. Si dans l'extrait analysé, l'enseignante " positive " à la fin (pas grave on va le faire maintenant) malgré tout, cette réflexion fait écho à de nombreux discours recueillis dans nos enquêtes (c'est épuisant de refaire sans cesse ce qu'on a déjà fait, on sait plus où on en est).

13. Ces propos transcrits ont été entendus lors de la séance observée mais ne se retrouvent pas tous dans les extraits longs de l'article.

14. Perceptible notamment au travers de l'intonation de l'enseignante ici.

15. Renvoie à un «non-gitan » en catalan-roussillonnais.

16. Enoncé en catalan-roussillonnais.

17. Propos entendus d'un des membres du personnel de la mairie de Perpignan.

18. Cf. reportage "Les enfants gitans du quartier Saint-Jacques à Perpignan » http:// www.dailymotion.com/video/xthxb8_les-enfants-gitans-du-quartier-saint-jacques-aperpignan_news.

19. L'enseignante montre aux élèves sur un livre une photo de l'artiste italien G. Arcimboldo (L'air). L'enfant voit sur un tableau un arbre là où l'enseignante voit un oiseau.

20. Ibid.

21. Nous n'entendons pas ici problématiser le singulier utilisé pour parler de manière englobante de «la» langue et de «la» culture des enfants sachant que bien entendu ces notions mériteraient une plus ample considération... 


\section{RÉSUMÉS}

Les spécificités du discours enseignant analysées ici proviennent d'un corpus recueilli dans une école accueillant exclusivement des enfants gitans. Les séances de classe étudiées renvoient à de nombreux discours tenus antérieurement que nous avons pu récolter dans le cadre d'une enquête ethnographique que nous avons menée durant une année (entre 2013 et 2014). Ces discours sont liés aux thèmes suivants : l'absentéisme, les langues et cultures en présence dans la classe, sujets étroitement corrélés à la scolarisation des enfants gitans. La notion de dialogisme nous a permis de comprendre, en acte, les répercussions de l'absentéisme sur les pratiques de classe et nous amène à développer différentes considérations didactiques.

We have collected data in in a school exclusively hosting gypsy children and analyzed teaching discourse in a classroom. The class sessions studied refer to many previous discourses that we were able to record during an ethnographic survey that we conducted previously. These teachers' discourses relate to the following subjects: absenteeism, languages and cultures in the classroom, subjects which are closely correlated with the schooling of gypsy children. The notion of dialogism has allowed us to understand, in action, the repercussions of absenteeism on class practices and led us to develop different didactic considerations.

\section{INDEX}

Mots-clés : dialogisme, analyse des interactions, élèves gitans

Keywords : dialogism, discourse analysis, gypsy pupils

\section{AUTEURS}

\section{NATHALIE AUGER}

Université Paul Valéry, Montpellier 3 - Praxiling, UMR 5267

Nathalie Auger est Professeur des universités en sciences du langage, linguistique et didactique du FLE/S, à l'Université Paul Valéry - Montpellier 3. Elle est chercheur au sein du laboratoire PRAXILING et responsable d'une équipe CNRS sur l'appropriation des langues et du langage. Elle s'occupe d'enseignements dans le cadre de la formation des futurs enseignants de français langue étrangère/langue non maternelle. Elle mène des recherches sur l'interculturel et le plurilinguisme en France, en Europe et au Canada. Elle prend en compte le multilinguisme et le multiculturalisme dans les cours de langue majoritaire et CLIL en Europe (projets Maledive et Conbat+ CELV, Conseil de l'Europe, 2011) et étudie les conditions de réussite des enfants gitans et roms dans les écoles françaises (Fonds social européen, Commission européenne, ortolang,

Erasmus + Romtels).

nathalie.auger [at] univ-montp3.fr 


\section{SOPHIE DUFOUR}

Université Paul Valéry, Montpellier 3 - Praxiling, UMR 5267

Sophie Dufour est Maître de conférences en didactique du FLE à l'Université Paul Valéry Montpellier 3. Ses travaux portent notamment sur les interactions didactiques et le français sur objectif universitaire. Elle mène des recherches sur la réception des cours magistraux, les représentations et pratiques universitaires des étudiants en mobilité.

sophie.dufour [at] univ-montp3.fr 